\title{
Short-distance constraints for the longitudinal component of the hadronic light-by-light amplitude: an update
}

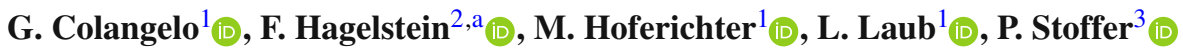 \\ ${ }_{1}$ Albert Einstein Center for Fundamental Physics, Institute for Theoretical Physics, University of Bern, Sidlerstrasse 5, 3012 Bern, Switzerland \\ 2 Paul Scherrer Institut, 5232 Villigen PSI, Switzerland \\ ${ }^{3}$ Faculty of Physics, University of Vienna, Boltzmanngasse 5, 1090 Vienna, Austria
}

Received: 5 July 2021 / Accepted: 29 July 2021 / Published online: 6 August 2021

(C) The Author(s) 2021

\begin{abstract}
We reassess the impact of short-distance constraints for the longitudinal component of the hadronic lightby-light amplitude on the anomalous magnetic moment of the muon, $a_{\mu}=(g-2)_{\mu} / 2$, by comparing different solutions that have recently appeared in the literature. In particular, we analyze the relevance of the exact axial anomaly and its impact on $a_{\mu}$ and conclude that it remains rather limited. We show that all recently proposed solutions agree well within uncertainties on the numerical estimate of the impact of shortdistance constraints on $a_{\mu}$, despite differences in the concrete implementation. We also take into account the recently calculated perturbative corrections to the massless quark loop to update our estimate and outline the path towards future improvements.
\end{abstract}

\section{Introduction}

The recent measurement of $(g-2)_{\mu}$ by the Fermilab Muon $g-2$ collaboration [1-4] has made the discrepancy with the Standard-Model prediction [5-29] more serious, by bringing it from the $3.7 \sigma$ to the $4.2 \sigma$ level when combined with the previous Brookhaven measurement [30]. The concrete perspective of additional reductions of the experimental uncertainty in the near future-mainly from subsequent Runs at Fermilab [31], but also the future J-PARC experiment [32] using a different technique-makes the need of further theoretical improvements more urgent. As is well known, the two main sources of theoretical uncertainties are both hadronic. The largest one is the hadronic vacuum polarization (HVP) contribution, and the second hadronic light-by-light (HLbL) scattering. A lot of work has been devoted to reducing the uncertainty in the latter by separately analyzing each of the different contributions to the HLbL amplitude. These involve

a e-mail: franziska.hagelstein@psi.ch (corresponding author) different intermediate states and their calculation requires a good understanding of the relevant physics. The present status has been summarized in the White Paper (WP) on the Standard Model prediction of $(g-2)_{\mu}$ [5], resulting in a phenomenological estimate in agreement with lattice QCD $[28,33]$. The behavior of the HLbL amplitude for asymptotic values of its arguments is fixed by QCD and represents an important, global constraint, which has a significant impact on the estimate of this contribution. A detailed understanding of which intermediate states play a role in satisfying this constraint is crucial to estimate its impact and reduce the overall theoretical uncertainty.

There are two different regimes of asymptotic momenta and correspondingly two different constraints. For $g-2$ kinematics, with one photon in the static limit, the HLbL amplitudes only depend on the squared momenta of the remaining three photons. The first regime is when two of them are much larger than the third, whereas all of them being about equally large defines the second. They represent two limiting cases of a possible continuum of short-distance constraints (SDCs) and we will refer to them as $\operatorname{SDC1}\left(q_{1,2}^{2} \gg q_{3}^{2}\right.$, $\left.q_{1,2}^{2} \gg \Lambda_{\mathrm{QCD}}^{2}\right)$ and $\operatorname{SDC} 2\left(q_{1}^{2} \sim q_{2}^{2} \sim q_{3}^{2} \gg \Lambda_{\mathrm{QCD}}^{2}\right)$. Melnikov and Vainshtein (MV) were the first to derive SDC1 [18] and, in particular, to point out that in the chiral limit and for asymptotic values of $q_{1,2}^{2}$, the leading $1 / q_{3}^{2}$ behavior of the longitudinal part receives no corrections neither at large nor at small $q_{3}^{2}$ values: in other words, the $1 / q_{3}^{2}$ dependence is exact across the whole range. This is a direct consequence of the axial anomaly [34-38], see Sect. 2. The transverse part in turn is constrained by the celebrated non-renormalization theorems $[39,40]$ for the vector-vector-axial-vector (VVA) correlator. The SDC2 case was also discussed by MV on the basis of the quark loop, but its derivation has only recently been put on a firm basis by using the operator product expansion (OPE) [25]. Moreover, both non-perturbative [41] and 
perturbative corrections [42] to the OPE have recently been calculated, thereby reducing one source of uncertainty.

There has been much interest in finding a way to satisfy these SDCs beyond the model solution discussed by MV [18]. We proposed a Regge model of pseudoscalar resonances [26,27], whereas a solution based on the resummation of a tower of axial-vector resonances in a holographic model of QCD (hQCD) was put forward in two more recent papers $[43,44]$. A completely different approach based on a set of interpolants between long and short distance has been adopted by Lüdtke and Procura (LP) [45]. Some of these works either appeared or were published after the WP, where the estimate about the impact of the SDCs and of the axial-vector contribution is significantly lower than what was estimated in [18]. While none of the most recent papers has criticized the estimate in the WP, there are statements in [44] that their results also agree with those in [18], and with [43], who in turn conclude that the MV model is not the correct way to implement SDC1, which makes the whole situation rather confusing. Given the relevance of the SDCs, which currently represent the largest contribution to the theoretical uncertainty of $a_{\mu}^{\mathrm{HLbL}}$ [5], it is important to understand the differences between these solutions, clarify to what extent they agree and where exactly differences arise, and reassess the current situation.

From a theoretical point of view, the solution based on hQCD is particularly relevant and appealing as it represents the first hadronic model of QCD based on axial vectors that exactly satisfies the axial anomaly and SDC1 in the chiral limit. As we will discuss in Sect. 3 on the basis of general, model-independent arguments, the solution has to arise from a resummation of an infinite tower of axial vectors, as it does in the hQCD models and as is expected when fulfilling SDCs with hadronic states $[46,47]$. The model also has the advantage that the resummation can be performed analytically, but its simplicity comes at the price of lack of flexibility: once a number of inputs is used to pin down the free parameters in the model, any further quantity can be predicted and shows some discrepancies with QCD phenomenology. In particular, in the simplest models on which we will focus here, the fulfillment of the asymptotic constraints in general generates tensions with phenomenological low-energy constraints [43,44].

The solution originally proposed by MV also exactly satisfies the axial anomaly and SDC1 in the chiral limit, but achieved this goal by a mere truncation: every hadronic contribution beyond the pion pole for $g-2$ kinematics was simply dropped. Such an approximation is very well justified for the three-point function $\langle V V A\rangle$, as explicitly shown in [44]. For what concerns the HLbL amplitude the situation is different: the MV model extrapolates the OPE expression to low $q_{1,2}^{2}$, where it cannot be justified. It was first pointed out in $[26,27]$ that the largest contribution to $a_{\mu}$ in the MV model comes from the low-energy region, where additional intermediate states would contribute. The first main point of this paper is then to demonstrate that this conclusion applies to all the recently proposed implementations, summarized in Sect. 4, explaining why there is general consensus on the numerical impact despite significant differences in the implementations themselves, see Sect. 5.

For instance, the hQCD and Regge models differ in their use of pseudoscalar vs. axial-vector states. In $[26,27]$ the main motivation for adopting a Regge model of pseudoscalar resonances is related to a peculiar property of their contribution to the HLbL tensor. In a dispersive approach [20,21,4851] the contribution of narrow-width resonances to the HLbL tensor is in general ambiguous as it depends on the basis in which the calculation is performed, unless a set of sum rules is satisfied. Only in the case of pseudoscalars are these sum rules automatically satisfied. The drawback of using pseudoscalar resonances is that in the chiral limit they do not couple to the axial current and therefore cannot contribute to the anomaly. We argued in $[26,27]$ that such a model would nonetheless represent a useful tool to make a realistic evaluation of the impact of the SDCs in the physical world, i.e., away from the chiral limit.

This view has been challenged by MV in [52]: they emphasized the importance of the axial anomaly as an exact constraint in the chiral limit, and considered its fulfillment essential in order to make a reliable estimate of the impact of the SDCs on $a_{\mu}$. By a detailed comparison between the MV and the hQCD model we will show, however, that the relevance of the exact axial anomaly in determining the four-point function is limited to a kinematic region whose impact on the calculation of $a_{\mu}^{\mathrm{HLbL}}$ is very small. This is one of the most important conclusions of this paper, which extends and confirms the findings in $[26,27]$, in line with earlier studies of the relevant momentum regions [53].

Since each of the models discussed here cannot claim to be a faithful representation of QCD but at best be a tool to capture the essential features thereof in connection with a particular aspect of the $a_{\mu}$ calculation, it is instructive to compare all three of them, even if they rely on different degrees of freedom to fulfill the SDCs. Anticipating our conclusions, we will find a satisfactory agreement between the hQCD and the pseudoscalar Regge models. We will then use the latter to update our earlier estimate taking into account the recently calculated perturbative and non-perturbative corrections to the OPE [41,42], see Sect. 6. This serves only to illustrate the current status, because we believe that it is possible to incorporate the good theoretical properties of hQCD models into our dispersive formalism, after developing a coherent formulation of axial vectors in the narrow-width approximation. This is the direction in which future work will evolve, as we will sketch in the outlook in Sect. 7. 


\section{The longitudinal OPE and non-renormalization theorems}

We concentrate here on the OPE for the longitudinal amplitude, which concerns only one of the functions in the HLbL tensor, namely the $\hat{\Pi}_{1}$ function introduced in [21]. Only this function contains the contribution of the pion pole, which can be written as ${ }^{1}$

$$
\begin{aligned}
\hat{\Pi}_{1}\left(q_{1}^{2}, q_{2}^{2}, q_{3}^{2}, 0 ; s, t, u\right)= & \frac{F_{\pi \gamma^{*} \gamma^{*}}\left(q_{1}^{2}, q_{2}^{2}\right) F_{\pi \gamma \gamma^{*}}\left(q_{3}^{2}\right)}{s-M_{\pi}^{2}} \\
& +\tilde{G}\left(q_{1}^{2}, q_{2}^{2}, q_{3}^{2}, 0 ; s, t, u\right) .
\end{aligned}
$$

The transition form factor (TFF) of the pion is a single function, which appears both in the doubly-virtual and in the singly-virtual case: $F_{\pi \gamma^{*} \gamma^{*}}\left(q^{2}, 0\right)=F_{\pi \gamma \gamma^{*}}\left(q^{2}\right)$. The function $\tilde{G}$ collects all additional contributions not containing any poles at $s=M_{\pi}^{2}$.

For the muon $g-2$ calculation we need to take the limit $q_{4} \rightarrow 0$, which changes the kinematics as follows: $s=q_{3}^{2}$, $t=q_{2}^{2}$, and $u=q_{1}^{2}$, leading to

$$
\begin{aligned}
\bar{\Pi}_{1}\left(q_{1}^{2}, q_{2}^{2}, q_{3}^{2}\right) \equiv & \hat{\Pi}_{1}\left(q_{1}^{2}, q_{2}^{2}, q_{3}^{2}, 0 ; q_{3}^{2}, q_{2}^{2}, q_{1}^{2}\right) \\
= & \frac{F_{\pi \gamma^{*} \gamma^{*}}\left(q_{1}^{2}, q_{2}^{2}\right) F_{\pi \gamma \gamma^{*}}\left(q_{3}^{2}\right)}{q_{3}^{2}-M_{\pi}^{2}} \\
& +G\left(q_{1}^{2}, q_{2}^{2}, q_{3}^{2}\right),
\end{aligned}
$$

where $G\left(q_{1}^{2}, q_{2}^{2}, q_{3}^{2}\right)=\tilde{G}\left(q_{1}^{2}, q_{2}^{2}, q_{3}^{2}, 0 ; q_{3}^{2}, q_{2}^{2}, q_{1}^{2}\right)$. We stress that taking the limit $q_{4} \rightarrow 0$ starting from the representation in (1) unambiguously leads to (2). The splitting between the first and second term is inherited from the splitting between pole term and the rest for general kinematics, but is nonetheless unique. If one wanted to identify the pole term directly for $g-2$ kinematics, the first term in (2) should only have its residue as numerator, thereby separating any additional $q_{3}^{2}$ dependence carried by the TFFs: in other words, separating the pseudoscalar poles from the vectormeson ones in the TFFs (which correspond to cuts from $2 \pi$, $3 \pi$, etc. intermediate states, if one does not take the narrowwidth approximation). Both definitions of the pion pole (for general or $g-2$ kinematics) are possible and the relation between the two is completely understood. The connection of this aspect with the SDCs has been discussed in detail in [54]. Here we adopt the splitting between pion pole and the function $G$ given in (2) and concentrate our discussion on the latter.

In the MV limit, $\hat{q}^{2} \equiv q_{1}^{2}=q_{2}^{2} \gg q_{3}^{2}, \hat{q}^{2} \gg \Lambda_{\mathrm{QCD}}^{2}$, with no constraints on $q_{3}^{2}$, the function $\bar{\Pi}_{1}$ reads:

\footnotetext{
${ }^{1}$ We work with $q_{4}^{2}=0$, but $q_{4} \neq 0$ and, for simplicity, only consider the isospin-triplet component for now. The Mandelstam variables are defined as $s=\left(q_{1}+q_{2}\right)^{2}, t=\left(q_{1}+q_{3}\right)^{2}, u=\left(q_{2}+q_{3}\right)^{2}$.
}

$$
\begin{aligned}
\bar{\Pi}_{1}\left(\hat{q}^{2}, \hat{q}^{2}, q_{3}^{2}\right)= & \frac{F_{\pi \gamma^{*} \gamma^{*}}\left(\hat{q}^{2}, \hat{q}^{2}\right) F_{\pi \gamma \gamma^{*}}\left(q_{3}^{2}\right)}{q_{3}^{2}-M_{\pi}^{2}} \\
& +G\left(\hat{q}^{2}, \hat{q}^{2}, q_{3}^{2}\right),
\end{aligned}
$$

which can be further simplified taking into account the leading-order OPE for the pion TFF [55,56]:

$$
F_{\pi \gamma^{*} \gamma^{*}}\left(\hat{q}^{2}, \hat{q}^{2}\right)=-\frac{2 F_{\pi}}{3 \hat{q}^{2}}+\mathcal{O}\left(\hat{q}^{-3}\right) .
$$

We now carry out the separation between the pion pole in $g-2$ kinematics (in the chiral limit) from the rest, and rewrite the expression for $\bar{\Pi}_{1}$ as follows:

$$
\begin{aligned}
\bar{\Pi}_{1}\left(\hat{q}^{2}, \hat{q}^{2}, q_{3}^{2}\right)= & -\frac{2 F_{\pi}}{3 \hat{q}^{2}}\left[\frac{F_{\pi \gamma \gamma}}{q_{3}^{2}}\right. \\
& \left.+\frac{F_{\pi \gamma \gamma^{*}}\left(q_{3}^{2}\right)-F_{\pi \gamma \gamma}}{q_{3}^{2}}+\mathcal{O}\left(M_{\pi}^{2}\right)\right] \\
& +G\left(\hat{q}^{2}, \hat{q}^{2}, q_{3}^{2}\right)+\mathcal{O}\left(\hat{q}^{-3}\right),
\end{aligned}
$$

where

$$
F_{\pi \gamma \gamma}:=F_{\pi \gamma \gamma^{*}}(0)=\frac{1}{4 \pi^{2} F_{\pi}} .
$$

Since we know how the amplitude has to behave in the chiral limit [18]:

$\left.\bar{\Pi}_{1}\left(\hat{q}^{2}, \hat{q}^{2}, q_{3}^{2}\right)\right|_{m_{q}=0}=-\frac{1}{6 \pi^{2}} \frac{1}{\hat{q}^{2} q_{3}^{2}}+\mathcal{O}\left(\hat{q}^{-3}\right)$,

we have to conclude that [27]

$$
\begin{aligned}
\left.G\left(\hat{q}^{2}, \hat{q}^{2}, q_{3}^{2}\right)\right|_{m_{q}=0}= & \left.\frac{2 F_{\pi}}{3 \hat{q}^{2}} \frac{F_{\pi \gamma \gamma^{*}}\left(q_{3}^{2}\right)-F_{\pi \gamma \gamma}}{q_{3}^{2}}\right|_{m_{q}=0} \\
& +\mathcal{O}\left(\hat{q}^{-3}\right) .
\end{aligned}
$$

This remarkable result is actually a consequence of the non-renormalization of the axial anomaly [34-38], as first discussed in [18] (see [39,40] for a full account of nonrenormalization theorems for the $V V A$ correlator):

$w_{L}\left(q_{1}^{2}, q_{2}^{2},\left(q_{1}+q_{2}\right)^{2}\right)=\frac{2 N_{C}}{\left(q_{1}+q_{2}\right)^{2}}+\mathcal{O}\left(M_{\pi}^{2}\right)$.

The expression on the right-hand side looks like the pion-pole contribution in the chiral limit, though without the (properly normalized) TFF in the numerator. As discussed in detail in $[54,57]$ this implies a constraint between the contribution of the pion and that of transverse degrees of freedom, such that the only effect of their contribution in the chiral limit is to replace the pion TFF by its value at $q_{3}^{2}=q_{4}^{2}=0$. A crucial point is that the constraint (8) applies for arbitrary values of 
$q_{3}^{2}$, but only for large values of $\hat{q}^{2} \gg \Lambda_{\mathrm{QCD}}^{2}\left(\right.$ and $\hat{q}^{2} \gg q_{3}^{2}$ ), where the OPE in the MV limit is valid: for non-asymptotic values of $q_{1,2}^{2}$ the connection between the four- and the threepoint function gets lost and nothing can be inferred on the behavior of $G\left(q_{1}^{2}, q_{2}^{2}, q_{3}^{2}\right)$. The key point in assessing the relevance of the non-renormalization theorem for $\langle V V A\rangle$ for the numerical evaluation of $a_{\mu}$ thus concerns the weight of the integration region in which the constraint applies, as we will discuss in detail in Sect. 5 .

\section{On axial-vector contributions to the longitudinal function in the dispersive approach}

Since the non-renormalization theorems on the $\langle V V A\rangle$ function interrelate transverse and longitudinal degrees of freedom, it is clear that axial-vector states play a role in fulfilling (8). Within the dispersive framework for HLbL, the inclusion of axial-vector mesons suffers from two closely related difficulties: on the one hand, the contribution of narrow states depends on the choice of basis - these ambiguities apply to all narrow resonances beyond the pseudoscalar ones and have been recently discussed for scalar contributions [58]. As only the full HLbL scattering amplitude needs to be basis independent, a phenomenological evaluation of axialvector effects thus must proceed in accordance with the other contributions. The axial-vector exchanges discussed in the context of SDCs, both in hQCD $[43,44]$ and in other implementations [18,52,57,59-61], typically refer to a Lagrangian model, which can differ by non-pole pieces from a dispersive definition, depending on the choice of basis. ${ }^{2}$

The second difficulty in the dispersive approach concerns kinematic singularities: while the basis of [21] is free from kinematic singularities in the dispersed Mandelstam variable, it still contains singularities in the photon virtualities, with residues that vanish for the entire HLbL contribution due to the presence of sum rules. As narrow resonances do not fulfill the sum rules individually, a further ambiguity in their contribution is introduced by the subtraction scheme of the singular parts, which again disappears only for the entire HLbL contribution. This second complication does not affect pseudoscalar or scalar contributions, but appears for axial and higher-spin resonances in the basis of [21]. By employing the sum rules, we have now constructed a new basis that explicitly removes all kinematic singularities from axial-vector contributions, while leaving pseudoscalar and

\footnotetext{
${ }^{2}$ Note that, in addition, all such estimates assume the validity of a narrow-width approximation. Since the main branching fractions proceed into three- or higher-multiplicity final states, a full dispersive treatment of axial-vector intermediate states is difficult, but for $S$ - and $D$-wave resonances that decay predominantly into two-meson states a comparison to implementations in terms of $\gamma^{*} \gamma^{*}$ amplitudes [62-69] is possible, see [58] for the scalar case.
}

scalar contributions unaltered, thereby solving this second issue in the case of axial-vector contributions. In this basis the contribution of a single axial-vector meson (with mass $M_{A}$ ) to the function $G$ takes the form:

$G\left(q_{1}^{2}, q_{2}^{2}, q_{3}^{2}\right)=\frac{G_{2}\left(q_{1}^{2}, q_{2}^{2}\right) G_{1}\left(q_{3}^{2}\right)}{M_{A}^{6}}$,

where

$$
\begin{aligned}
G_{2}\left(q_{1}^{2}, q_{2}^{2}\right)= & \left(q_{1}^{2}-q_{2}^{2}\right) \mathcal{F}_{1}\left(q_{1}^{2}, q_{2}^{2}\right) \\
& +q_{1}^{2} \mathcal{F}_{2}\left(q_{1}^{2}, q_{2}^{2}\right)+q_{2}^{2} \mathcal{F}_{2}\left(q_{2}^{2}, q_{1}^{2}\right) \\
G_{1}\left(q^{2}\right)= & \mathcal{F}_{1}\left(q^{2}, 0\right)+\mathcal{F}_{2}\left(q^{2}, 0\right)=\frac{G_{2}\left(q^{2}, 0\right)}{q^{2}}
\end{aligned}
$$

and $\mathcal{F}_{1,2}\left(q_{1}^{2}, q_{2}^{2}\right)$ are two of the three TFFs of an axial-vector meson, see [70] for the precise definitions. The third one, $\mathcal{F}_{3}$, does not appear in the expression above but is related to $\mathcal{F}_{2}$ by the symmetry properties of the TFFs:

$\mathcal{F}_{1}\left(q_{1}^{2}, q_{2}^{2}\right)=-\mathcal{F}_{1}\left(q_{2}^{2}, q_{1}^{2}\right)$,

$\mathcal{F}_{2}\left(q_{2}^{2}, q_{1}^{2}\right)=-\mathcal{F}_{3}\left(q_{1}^{2}, q_{2}^{2}\right)$.

The expression (11) shows that the dispersive contribution of axial-vector mesons to the function $G$ has the form of nonpole terms, but does not vanish. Our new basis avoids any kinematic singularities in (10) and makes the dependence on the virtualities unambiguous for basis changes that preserve this property, up to terms that are subleading for $q_{i}^{2} \gg M_{A}^{2}$.

As the remaining ambiguities become irrelevant for asymptotic virtualities, (10) leads to an interesting modelindependent conclusion. The light-cone expansion determines the asymptotic behavior of $\mathcal{F}_{1}=\mathcal{O}\left(1 / q_{i}^{6}\right), \mathcal{F}_{2}=$ $\mathcal{O}\left(1 / q_{i}^{4}\right)$, with coefficients determined via decay constants in analogy to (4), see [70]. This implies that, asymptotically,

$G_{2}\left(\hat{q}^{2}, \hat{q}^{2}\right)=\mathcal{O}\left(1 / \hat{q}^{2}\right), \quad G_{1}\left(q_{3}^{2}\right)=\mathcal{O}\left(1 / q_{3}^{4}\right)$.

Since (10) factorizes into parts dependent on $q_{1,2}^{2}$ and $q_{3}^{2}$, respectively, we find that (8) decomposes into two equations that need to be fulfilled separately:

$$
\begin{aligned}
\lim _{\hat{q}^{2} \rightarrow \infty} x \frac{G_{2}\left(\hat{q}^{2}, \hat{q}^{2}\right)}{M_{A}^{4}} & =-\frac{2}{3 \hat{q}^{2}}+\mathcal{O}\left(\hat{q}^{-3}\right), \\
\frac{G_{1}\left(q_{3}^{2}\right)}{x M_{A}^{2}} & =-F_{\pi} \frac{F_{\pi \gamma \gamma^{*}}\left(q_{3}^{2}\right)-F_{\pi \gamma \gamma}}{q_{3}^{2}},
\end{aligned}
$$

with $x$ an unknown, but constant factor that depends on the axial-vector TFFs. While the asymptotic form of $G_{2}$ thus matches, the axial-vector contribution to $G_{1}\left(q_{3}^{2}\right)$ decreases 


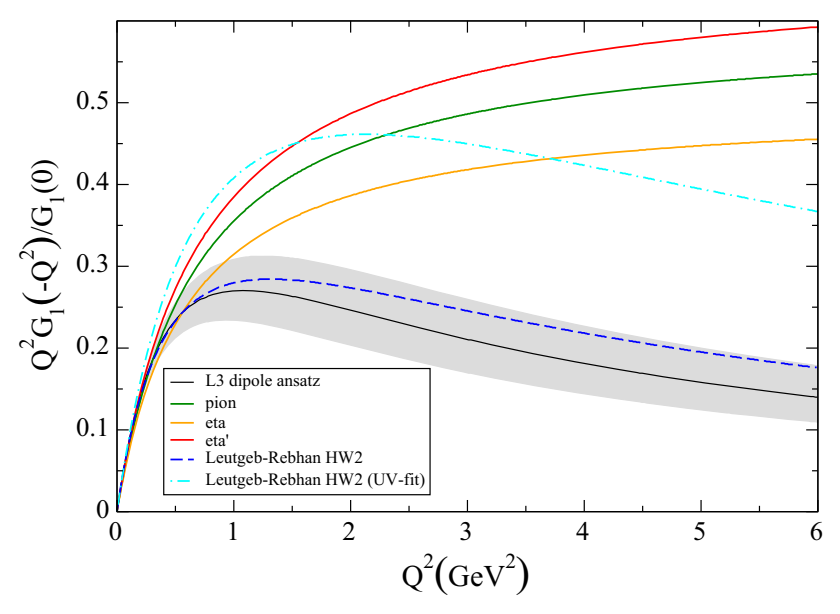

Fig. 1 Singly-virtual TFF of the ground-state axial-vector meson $f_{1}(1285)$ : comparison of the dipole ansatz used to fit the L3 data (black curve and gray band) [71], the HW2 hQCD model representations (blue dashed and turquoise dot-dashed curves) [43], and the one obtained from (14) using as input the $\pi^{0}, \eta$, and $\eta^{\prime}$ TFFs (green, yellow, and red curves) from [27]

too fast, mirroring the need for an infinite tower of axialvector states in the hQCD models. This mismatch can also be illustrated by comparing $Q^{2} G_{1}\left(-Q^{2}\right) / G_{1}(0)$ evaluated from the axial-vector TFFs with (14), see Fig. 1, where we concentrated on the contribution from $\mathcal{F}_{2}$, given that $\mathcal{F}_{1}$ is suppressed for several reasons: for small virtualities its antisymmetry implies $\mathcal{F}_{1}\left(-Q^{2}, 0\right) \sim Q^{2}$, for large virtualities due to the asymptotic behavior, and phenomenologically due to small couplings [72]. The comparison curves for $\eta$ and $\eta^{\prime}$ show that this qualitative behavior does not depend on the isospin channel, reinforcing that a single state is not sufficient to implement the SDCs.

\section{Three approaches to satisfy short-distance constraints}

In this section we compare the different solutions to the SDCs that have been proposed so far in the literature $[18,26,27$, $43,44,52]$, in terms of the different representations of the functions $w_{L}\left(q^{2}\right) \equiv w_{L}\left(q^{2}, 0, q^{2}\right)$ and $G\left(q_{1}^{2}, q_{2}^{2}, q_{3}^{2}\right)$, as constrained by the non-renormalization theorem (9) and the asymptotic behavior (8) in the chiral limit. We consider the models as they are, in other words, we take each one of them as an approximation to the total contribution to $w_{L}$ and the longitudinal SDC. The question of building a better model, possibly by combining features or degrees of freedom of the present ones will be touched upon in Sect. 7. The analysis based on interpolants [45] will be included in the numerical comparison in the following section.

\subsection{The Melnikov-Vainshtein model}

After deriving the SDC for the HLbL tensor, MV go beyond the asymptotic limit and propose a model that by construction satisfies (7):

$\bar{\Pi}_{1}^{\mathrm{MV}}\left(q_{1}^{2}, q_{2}^{2}, q_{3}^{2}\right)=\frac{F_{\pi \gamma^{*} \gamma^{*}}\left(q_{1}^{2}, q_{2}^{2}\right) F_{\pi \gamma \gamma}}{q_{3}^{2}-M_{\pi}^{2}}$,

with the shift in the pole position from zero to $M_{\pi}^{2}$ as the only effect of the light quark masses considered. This implies that, even though no additional contributions beyond the pion pole are introduced explicitly, such additional contributions are implicitly assumed to be completely determined not only in the asymptotic region, as implied by (8), but everywhere:

$$
\begin{aligned}
G^{\mathrm{MV}}\left(q_{1}^{2}, q_{2}^{2}, q_{3}^{2}\right)= & -F_{\pi \gamma^{*} \gamma^{*}}\left(q_{1}^{2}, q_{2}^{2}\right) \frac{F_{\pi \gamma \gamma^{*}}\left(q_{3}^{2}\right)-F_{\pi \gamma \gamma}}{q_{3}^{2}} \\
& +\mathcal{O}\left(M_{\pi}^{2}\right)
\end{aligned}
$$

Equation (16) is a very strong assumption, with no apparent physical justification: it extrapolates a constraint only valid at asymptotically high energies (8) to all possible values of $q_{1,2}^{2}$, all the way down to $q_{1}^{2}=q_{2}^{2}=0$. As such, it has the potential to significantly affect the value of the HLbL contribution to $a_{\mu}$ - a quantity most sensitive to low $q_{i}^{2}$. That this indeed happens has already been shown explicitly in [27], and will be discussed in more detail below.

Regarding the three-point function, the MV model reads

$w_{L}^{\mathrm{MV}}\left(q^{2}\right)=\frac{2 N_{C}}{q^{2}-M_{\pi}^{2}}$,

which again amounts to including as only chiral correction the one that shifts the pole in the pion propagator. Since chiral corrections become negligible at large $q^{2},(17)$ is a wellmotivated model at all $q^{2}$, with small deviations from the truth expected only at intermediate energies (chiral corrections may become more sizable for the $\eta$ and $\eta^{\prime}$ channels).

\subsection{The Leutgeb-Rebhan and \\ Cappiello-Catà-D'Ambrosio-Greynat-Iyer models}

In two recent papers, Leutgeb-Rebhan [43] (LR) and Cappiello et al. [44] (CCDGI) have proposed models based on hQCD to satisfy the SDCs. As the discussion in Sect. 3 shows, a solution in terms of a single axial-vector meson (per isospin channel) is essentially excluded, and indeed in these models the solution emerges from a resummation of an infinite tower of axial-vector mesons. For simplicity, we concentrate here on the model presented by CCDGI in [44], which is equivalent to the HW2 model in [43], although the two groups 
make different choices for the parameters. ${ }^{3}$ The representation of this model for the function $G$ reads (in the notation of CCDGI)

$$
\begin{aligned}
G^{\mathrm{HW} 2}\left(q_{1}^{2}, q_{2}^{2}, q_{3}^{2}\right)= & -\frac{F_{\pi \gamma^{*} \gamma^{*}}\left(q_{1}^{2}, q_{2}^{2}\right) F_{\pi \gamma \gamma^{*}}\left(q_{3}^{2}\right)}{q_{3}^{2}} \\
& -\frac{F_{\pi \gamma \gamma}^{2}}{q_{3}^{2}} \int_{0}^{z_{0}} d z \alpha^{\prime}(z) v_{1}(z) v_{2}(z) v_{3}(z),
\end{aligned}
$$

where $\alpha(z)=1-z^{2} / z_{0}^{2}$, with $z_{0}=\left(\sqrt{2} \pi F_{\pi}\right)^{-1}$ and

$v_{i}(z)=z Q_{i}\left[K_{1}\left(z Q_{i}\right)+\frac{K_{0}\left(z_{0} Q_{i}\right)}{I_{0}\left(z_{0} Q_{i}\right)} I_{1}\left(z Q_{i}\right)\right]$,

with $K_{n}(x)$ and $I_{n}(x)$ modified Bessel functions [44]. The same function $v_{i}(z)$ also determines the pion TFF

$F_{\pi \gamma^{*} \gamma^{*}}\left(q_{1}^{2}, q_{2}^{2}\right)=-F_{\pi \gamma \gamma} \int_{0}^{z_{0}} d z \alpha^{\prime}(z) v_{1}(z) v_{2}(z)$.

This representation correctly reproduces the high- $q^{2}$ limit of the TFF shown in (4), the Brodsky-Lepage limit of the singly-virtual pion TFF $[55,56]$ :

$F_{\pi \gamma \gamma^{*}}\left(\hat{q}^{2}\right)=-\frac{2 F_{\pi}}{\hat{q}^{2}}+\mathcal{O}\left(\hat{q}^{-3}\right)$,

as well as, by construction, the normalization at $q_{1}^{2}=q_{2}^{2}=0$. A convenient rewriting for $G$ is

$$
\begin{aligned}
G^{\mathrm{HW} 2}\left(q_{1}^{2}, q_{2}^{2}, q_{3}^{2}\right)= & -F_{\pi \gamma^{*} \gamma^{*}}\left(q_{1}^{2}, q_{2}^{2}\right) \frac{F_{\pi \gamma \gamma^{*}}\left(q_{3}^{2}\right)-F_{\pi \gamma \gamma}}{q_{3}^{2}} \\
& -\frac{F_{\pi \gamma \gamma}^{2}}{q_{3}^{2}} \int_{0}^{z_{0}} d z \alpha^{\prime}(z) v_{1}(z) v_{2}(z) \bar{v}_{3}(z),
\end{aligned}
$$

where $\bar{v}_{3}(z)=v_{3}(z)-1$, as it shows that there is no divergence at $q_{3}^{2}=0$ (the integral vanishes for $q_{3}^{2} \rightarrow 0$ ). Note that the first term in (22) coincides, up to chiral corrections, with the MV model, which can therefore be viewed as a truncated version of the hQCD model. In the HW2 model, however, the first and the second non-factorizable term always come together as they have the same physical origin: both arise from the resummation of the whole tower of axial-vector mesons. In the numerical analysis below we will see that, while the first term is dominant for asymptotic values of $q_{1}^{2} \sim q_{2}^{2}$, for low momenta, they are equally important and in fact cancel each other.

\footnotetext{
3 We will not consider the HW1 model discussed in [43] simply because it does not offer a simple analytic representation like HW2.
}

We also observe that the HW2 model offers a compact and convenient representation for the function $\bar{\Pi}_{1}$ :

$$
\begin{aligned}
\bar{\Pi}_{1}^{\mathrm{HW} 2}= & F_{\pi \gamma^{*} \gamma^{*}}\left(q_{1}^{2}, q_{2}^{2}\right) \frac{F_{\pi \gamma \gamma}}{q_{3}^{2}-M_{\pi}^{2}} \\
& \times\left[1+\frac{M_{\pi}^{2}\left(F_{\pi \gamma \gamma^{*}}\left(q_{3}^{2}\right)-F_{\pi \gamma \gamma}\right)}{q_{3}^{2} F_{\pi \gamma \gamma}}\right] \\
& -\frac{F_{\pi \gamma \gamma}^{2}}{q_{3}^{2}} \int_{0}^{z_{0}} d z \alpha^{\prime}(z) v_{1}(z) v_{2}(z) \bar{v}_{3}(z),
\end{aligned}
$$

where one can clearly see that the corrections to the $1 / q_{3}^{2}$ behavior, i.e., the pion pole in $g-2$ kinematics, vanish in the chiral limit-the integral behaves as $\mathcal{O}\left(\hat{q}^{-4}\right)$.

Finally, for the $V V A$ correlation function the HW2 model gives

$w_{L}^{\mathrm{HW} 2}\left(q^{2}\right)=\frac{2 N_{C}}{q^{2}-M_{\pi}^{2}}\left[1+\frac{M_{\pi}^{2}\left(F_{\pi \gamma \gamma^{*}}\left(q^{2}\right)-F_{\pi \gamma \gamma}\right)}{q^{2} F_{\pi \gamma \gamma}}\right]$,

which shows that the first term again corresponds to the MV model. In this case it is evident that the corrections to the MV model are of $\mathcal{O}\left(M_{\pi}^{2}\right)$ for any value of $q^{2}$, and therefore expected to be small everywhere. This expression also shows that the first term in the representation of the four-point function (23) takes the form $F_{\pi \gamma^{*} \gamma^{*}}\left(q_{1}^{2}, q_{2}^{2}\right) F_{\pi \gamma \gamma} w_{L}\left(q_{3}^{2}\right) /\left(2 N_{C}\right)$. While there is no harm in approximating the $w_{L}$ function with (17), it is dropping the non-factorizable second term in (23) that amounts to an uncontrolled approximation. Its numerical impact will be shown in Sect. 5.

\subsection{Regge model of excited pseudoscalars}

In the model we presented in $[26,27]$, we considered only the contribution of excited pseudoscalars to the function $G$ :

$G^{\mathrm{eP}}\left(q_{1}^{2}, q_{2}^{2}, q_{3}^{2}\right)=\sum_{i=1}^{\infty} \frac{F_{P_{i} \gamma^{*} \gamma^{*}}\left(q_{1}^{2}, q_{2}^{2}\right) F_{P_{i} \gamma \gamma^{*}}\left(q_{3}^{2}\right)}{q_{3}^{2}-M_{P_{i}}^{2}}$.

Clearly, by dropping axial-vector intermediate states, which contribute to this function according to (10), we are transferring their unique role in the chiral limit to the pseudoscalars, which amounts to effectively changing their chiral behavior, in particular the coupling of the excited pseudoscalars to the axial-vector current, which has to vanish in the chiral limit. This procedure cannot be strictly justified, but is similar in spirit to models that use constituent quark masses. In order to remove some of the model dependence, after matching to the behavior dictated by the OPE we are replacing our model 


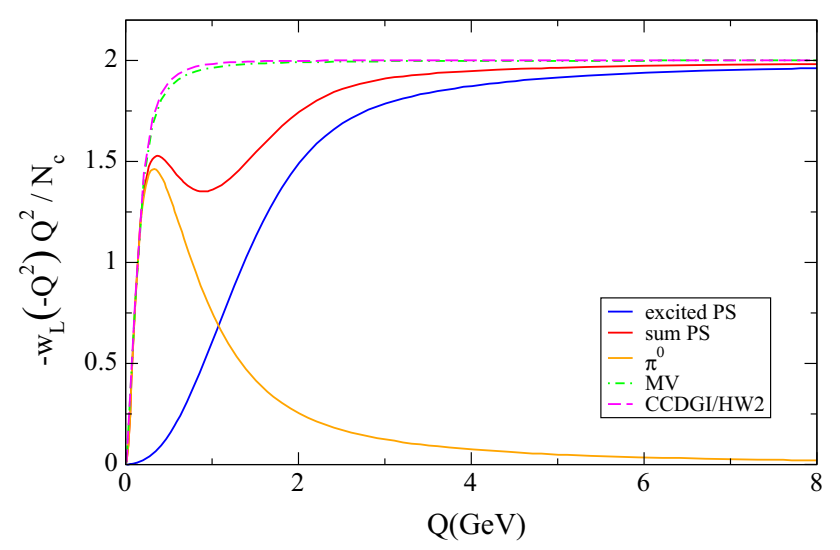

Fig. 2 Comparison of the MV, the HW2, and our model (solid) for the isovector component of $-Q^{2} w_{L}\left(-Q^{2}\right)$ as a function of $Q$. Our model is also broken down into pion and sum of excited pseudoscalar contributions

in the asymptotic region with the perturbative QCD quark loop.

We have imposed as constraint to our model that it satisfy (8) only for $q_{3}^{2} \gg \Lambda_{\mathrm{QCD}}$, which is a less ambitious goal than the one reached by both models described above:

$\lim _{\hat{q}^{2} \rightarrow \infty} \hat{q}^{2} G^{\mathrm{eP}}\left(\hat{q}^{2}, \hat{q}^{2}, q_{3}^{2}\right)=-\frac{1}{6 \pi^{2} q_{3}^{2}}+\mathcal{O}\left(q_{3}^{-3}\right)$.

By construction, our model takes into account singularities that are known to be present in the spectrum of QCD (the low-lying pseudoscalar excitations). The resummation of all higher excitations is used essentially to achieve the matching to the asymptotic behavior, but its precise form is inessential.

The resulting representation for the longitudinal component of the $V V A$ correlator becomes ${ }^{4}$

$w_{L}^{\mathrm{eP}}\left(q^{2}\right)=\frac{2 N_{C}}{F_{\pi \gamma \gamma}}\left[\frac{F_{\pi \gamma \gamma^{*}}\left(q^{2}\right)}{q^{2}-M_{\pi}^{2}}+\sum_{i=1}^{\infty} \frac{F_{P_{i} \gamma \gamma^{*}}\left(q^{2}\right)}{q^{2}-M_{P_{i}}^{2}}\right]$.

We stress that this model was not conceived to approximate this function other than for asymptotic values of its argument, and its use in [26,27] was limited to the four-point function. However, we find it instructive to provide this expression and compare it numerically to the other models.

\section{Numerical comparison of the three models}

In this section we compare the three models numerically, first for the $w_{L}\left(q^{2}\right)$ function, then the $G$ function and its contribution to $a_{\mu}$.

${ }^{4}$ Note that here we are using a peculiarity of our model that $F_{P_{i} \gamma^{*} \gamma^{*}}\left(\hat{q}^{2}, \hat{q}^{2}\right)=-\frac{1}{6 \pi^{2} F_{\pi \gamma \gamma} \hat{q}^{2}}+\mathcal{O}\left(\hat{q}^{-3}\right)$ for all $i$.

\subsection{The $w_{L}$ function}

A numerical comparison between the hQCD model and the MV model for the $w_{L}$ function was provided and discussed in [44] and clearly showed that the difference between the two models amounts to chiral corrections neglected by MV, which the hQCD model estimates to be numerically very small. The picture that emerges from the comparison is that the $w_{L}$ function is essentially determined by its low-energy (fixed by the pion pole) and its high-energy behavior, with no room for any structure in between. In Fig. 2 we repeat the comparison for the isovector channel and show in addition the contribution of the pion if one includes its transition form factor in the numerator-in other words, according to the dispersive definition of the pion contribution for general kinematics. The difference between the $\pi^{0}$ and the CCDGI/HW2 curves is the contribution of the axials, but its main effect is to remove the TFF from the numerator, as the minute difference to the MV model shows. The hQCD models confirm that the MV model appears to be an excellent approximation to the true $w_{L}$ function in QCD. It is instructive to see algebraically how the contribution of the axial-vector states manages to remove the TFF from the pion-pole contribution and also to be able to estimate the additional corrections to it, but for all practical purposes, and unless the highest precision is required, the MV model seems to provide an excellent description of $w_{L}$. In the same plot we also show our model of excited pseudoscalars. This is designed to agree with the other two for asymptotic values of $q^{2}$, as indeed it does. At low energy, where the pion contribution dominates, it also agrees with the other two, but the transition region is not as smooth and shows some structure, with up to $30 \%$ discrepancy with the other two models. There is nothing to be read into this discrepancy other than the fact that the model was never designed to provide a good description of $w_{L}$ : the structure it shows in the intermediate region just reflects the fact that it was not required to fulfill any constraints here. If required, the model could be refined to improve the transition between low- and high-energy constraints. Whether this is of relevance in the context of the four-point function will be discussed in the following.

\subsection{The $G$ function and the role of $w_{L}$}

Such a good understanding of the function $w_{L}$ and the simple and accurate description provided by the MV model raises the question which role the three-point function plays in determining the four-point function: how strongly does the accurate knowledge of $w_{L}$ constrain $\bar{\Pi}_{1}$ or $G$ ? MV have shown that for asymptotic values of $q_{1}^{2} \sim q_{2}^{2}$, the leading behavior of $G$ is completely fixed by $w_{L}$, but how relevant is the asymptotic region in determining the contribution of $G$ to $a_{\mu}$ ? In [52] it is argued that the kinematic region $q_{1}^{2} \sim q_{2}^{2} \gg q_{3}^{2}$ 


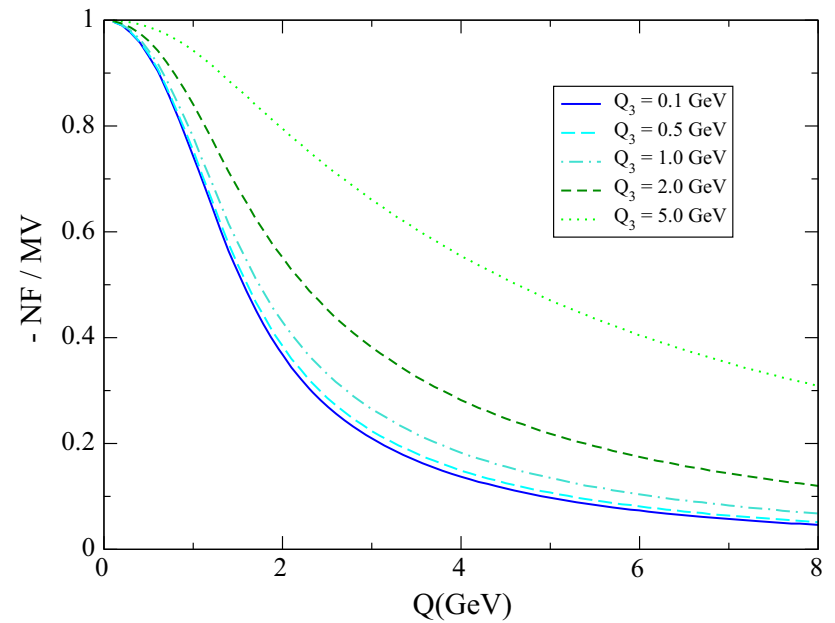

Fig. 3 Comparison between the non-factorizable (NF) and the MV term (second and first term in (22), respectively, for $q_{1}^{2}=q_{2}^{2}=-Q^{2}$ and $q_{3}^{2}=-Q_{3}^{2}$ ) in the CCDGI model (set 2)

"provides the largest contribution to $a_{\mu}^{\mathrm{HLbL}}$," but we are not aware of any quantitative basis for such a statement. When we proposed an alternative way to fulfill the SDCs and compared to MV [26,27], we showed that the large difference between ours and the MV model arose precisely in the low- $q_{i}^{2}$ region and, moreover, that the largest contribution to $a_{\mu}^{\mathrm{HLbL}}$ in the MV model itself came from the same region.

The hQCD models, which satisfy exactly the anomaly and the MV constraints, allow us to test the approximation made in the MV model in a more quantitative way. As discussed above, there are two approximations made by MV in their representation of $\bar{\Pi}_{1}$ : the first is to neglect chiral correction in $w_{L}\left(q_{3}^{2}\right)$, which is a very good one as we have just seen, but the second one is to neglect non-factorizable corrections, which in the hQCD models are given by the integral term in (23). To establish the relevance of the function $w_{L}$ in the calculation of the four-point function and its contribution to $a_{\mu}$ we can therefore compare the non-factorizable and the MV term: the region where the latter dominates is the region where the MV limit matters, i.e., where the $w_{L}$ function plays an important role, and the MV model is a good approximation. This comparison is shown in Fig. 3: even for the modest requirement that the non-factorizable term amounts to at most $30 \%$ of the MV term, the minimum value of $Q$ for which this is satisfied is above $2 \mathrm{GeV}$. At the matching point $Q_{\text {match }}=$ $1.7 \mathrm{GeV}$, adopted in $[26,27]$ for the transition between the hadronic and the pQCD description, and which will again be used below, the non-factorizable term is at least a $50 \%$ correction to the MV term.

This suggests that if we compare the MV and the hQCD models at the level of the function $G$, the agreement is going to be much worse than for the function $w_{L}$. To verify this expectation, we plot the isovector component of the function

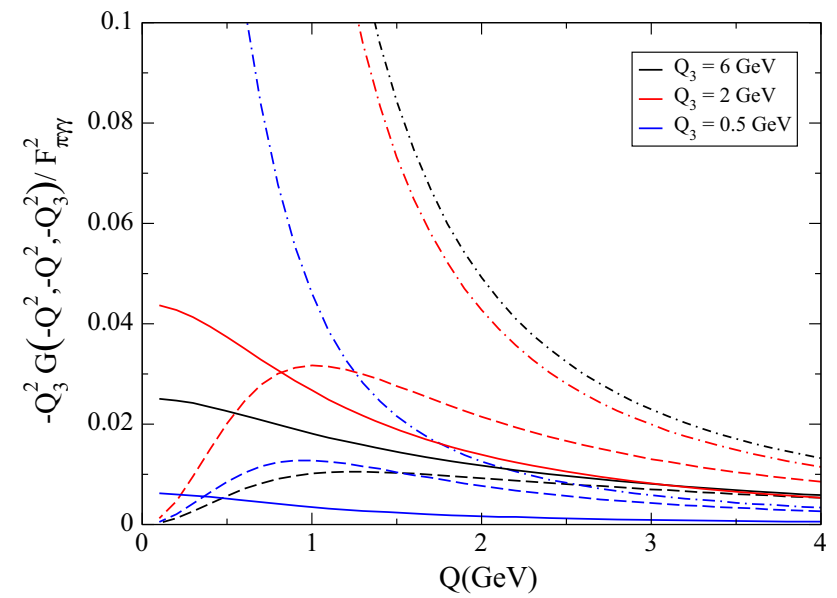

Fig. 4 Comparison of the MV (dot-dashed), the HW2 (dashed), and our model (solid) for $-Q_{3}^{2} G\left(-Q^{2},-Q^{2},-Q_{3}^{2}\right) / F_{\pi \gamma \gamma}^{2}$ as a function of $Q$ for different values of $Q_{3}$

$-Q_{3}^{2} G\left(-Q^{2},-Q^{2},-Q_{3}^{2}\right)$ as a function of $Q^{2}$, see Fig. 4 . The dashed curves show the HW2 model, whereas the dotdashed ones only show the first term of (22), which corresponds to the MV model. The plot shows very clearly that the latter two versions tend to the same asymptotic limit, even for low values of $Q_{3}$, but that they differ significantly at low $Q^{2}$. While at large $Q^{2}$ the second term in (22) is subdominant and can be neglected, it compensates exactly the first one for low $Q^{2}$, so that their sum vanishes. This is expected for axialvector mesons, and is already seen in Fig. 4. If one keeps just the first term, i.e., the MV model, this grows at low $Q^{2}$, reaching a finite limit for $Q^{2}=0$ (not visible in the plot range, because it is quite large). Figure 4 also displays our model (solid curves), which has non-vanishing limits for $Q^{2}=0$, too. This is well understood, however, because excited pseudoscalars do couple to two real photons (even in the chiral limit). As detailed in [27], these couplings are compatible with the available phenomenological information, which still suffers from large uncertainties. By construction, our model also agrees with the other two for large $Q^{2}$ and $Q_{3}^{2}$, whereas for low $Q_{3}^{2}$ it does not agree well with the other two even as $Q^{2}$ grows.

\subsection{Contribution to $a_{\mu}$ of the function $G$}

We can now address the question of how these differences are reflected in the calculation of the contribution to $a_{\mu}$. We do so by breaking down the contributions from different kinematic regions and separating the isovector channel from the isoscalar and isosinglet ones. Identifying the isoscalar and isosinglet pieces with the physical states ignores mixing effects, which implies that the $\eta / f_{1}$ and $\eta^{\prime} / f_{1}^{\prime}$ cannot be compared separately. In general, the correct implementation of mixing effects requires two mixing angles (see [73] for a 
Table 1 Contribution of $G$ to $a_{\mu}$ (referred to as the longitudinal SD contribution in $[26,27]$ and the longitudinal axial-vector contribution in $[43,44])$ from the isovector and isoscalar plus isosinglet channels broken down in different integration regions $\left(Q_{\text {match }}=1.7 \mathrm{GeV}\right)$. The notation for the mixed regions includes the respective crossed versions, e.g., the second line gives the contribution from $\bar{\Pi}_{1}$ in the region $Q_{1,2}^{2}>Q_{\text {match }}^{2}>Q_{3}^{2}$ and from $\bar{\Pi}_{2}$ in the region $Q_{1,3}^{2}>Q_{\text {match }}^{2}>Q_{2}^{2}$, in such a way that the region in which the SDC1 applies is contained in this (and partly the first) row, while the third row has a scaling $1 / Q^{4}$ in the hard momenta. Due to different mixing patterns the $\eta / f_{1}$ and $\eta^{\prime} / f_{1}^{\prime}$ contributions cannot be compared separately. Note that the Reggemodel contribution to the asymptotic region is not yet replaced by the OPE result. The numbers for LP refer to the "reference interpolant" of [45]. The HW1 model, which we have not considered here, gives a higher contribution $\Delta a_{\mu}=23.2 \times 10^{-11}$ [43]. All entries are understood to be accurate at the level of \pm 0.1 due to the applied numerical integration methods, other (model-dependent) errors are not shown

\begin{tabular}{|c|c|c|c|c|c|c|c|}
\hline & \multirow[t]{2}{*}{ MV } & \multicolumn{2}{|c|}{ CCDGI } & \multicolumn{2}{|l|}{$\underline{\mathrm{LR}}$} & \multirow[t]{2}{*}{ LP } & \multirow[t]{2}{*}{ PS Regge } \\
\hline & & set 1 & set 2 & HW2 & HW2UV-fit & & \\
\hline & \multicolumn{7}{|c|}{$\Delta a_{\mu}^{\pi / a_{1}} \times 10^{11}$} \\
\hline$\overline{Q_{i}^{2}>Q_{\text {match }}^{2} \forall i}$ & 1.4 & 0.5 & 0.8 & 0.6 & 0.8 & 0.9 & 0.7 \\
\hline$Q_{1,2}^{2}>Q_{\text {match }}^{2}>Q_{3}^{2}$ & 1.4 & 0.8 & 1.0 & 0.8 & 1.0 & 0.3 & 0.4 \\
\hline$Q_{i, 3}^{2}>Q_{\text {match }}^{2}>Q_{j}^{2} \quad i \neq j \neq 3$ & 0.8 & 0.2 & 0.3 & 0.2 & 0.3 & 0.4 & 0.4 \\
\hline$Q_{i}^{2}>Q_{\text {match }}^{2}>Q_{j, k}^{2} \quad i \neq j \neq k$ & 0.8 & 0.3 & 0.4 & 0.3 & 0.4 & 0.3 & 0.2 \\
\hline$Q_{\text {match }}^{2}>Q_{i}^{2} \quad \forall i$ & 11.8 & 2.2 & 1.7 & 2.3 & 1.8 & 0.7 & 1.0 \\
\hline \multirow[t]{2}{*}{ Total } & 16.2 & 4.0 & 4.2 & 4.2 & 4.3 & 2.6 & 2.7 \\
\hline & \multicolumn{7}{|c|}{$\Delta a_{\mu}^{\eta / f_{1}+\eta^{\prime} / f_{1}^{\prime}} \times 10^{11}$} \\
\hline$\overline{Q_{i}^{2}>Q_{\text {match }}^{2} \forall i}$ & 3.4 & 1.4 & 1.7 & 1.7 & 2.5 & 2.5 & 3.1 \\
\hline$Q_{1,2}^{2}>Q_{\text {match }}^{2}>Q_{3}^{2}$ & 2.1 & 2.1 & 2.3 & 2.5 & 3.0 & 0.6 & 1.1 \\
\hline$Q_{i, 3}^{2}>Q_{\text {match }}^{2}>Q_{j}^{2} \quad i \neq j \neq 3$ & 1.9 & 0.6 & 0.7 & 0.6 & 0.9 & 1.2 & 1.6 \\
\hline$Q_{i}^{2}>Q_{\text {match }}^{2}>Q_{j, k}^{2} \quad i \neq j \neq k$ & 1.7 & 0.8 & 0.9 & 0.9 & 1.1 & 0.7 & 0.9 \\
\hline$Q_{\text {match }}^{2}>Q_{i}^{2} \quad \forall i$ & 12.9 & 5.6 & 5.1 & 6.8 & 5.4 & 1.5 & 3.1 \\
\hline Total & 22.1 & 10.4 & 10.7 & 12.5 & 12.8 & 6.5 & 9.9 \\
\hline$\overline{\text { Grand total }\left(\pi / a_{1}+\eta / f_{1}+\eta^{\prime} / f_{1}^{\prime}\right)}$ & 38.3 & 14.3 & 14.9 & 16.7 & 17.1 & 9.1 & 12.6 \\
\hline
\end{tabular}

review), but the differences can be illustrated based on the simple $\mathrm{U}(3)$ formula

$$
\frac{\Gamma(P \rightarrow \gamma \gamma)}{\Gamma\left(P^{\prime} \rightarrow \gamma \gamma\right)}=\frac{M_{P}}{M_{P^{\prime}}} \cot ^{2}\left(\theta_{A}-\theta_{0}\right), \theta_{0}=\arcsin \frac{1}{3},
$$

which for $P=f_{1}$ gives $\theta_{A}=62(5)^{\circ}$ [71,74], but $\theta_{A}=$ $84.8(6)^{\circ}$ for $P=\eta$. For this reason, we only compare the sum of $\eta$ and $\eta^{\prime}$ with the sum of $f_{1}$ and $f_{1}^{\prime}$ contributions, which are not affected by this ambiguity. Table 1 shows that, although the CCGDI/HW2 and our model completely differ in the degrees of freedom that are used to satisfy the relevant SDCs, they give similar numerical contributions to $a_{\mu}$. The MV model, which satisfies (8) exactly, much like CCGDI/HW2, but by neglecting any contribution to $\bar{\Pi}_{1}$ beyond the pion pole in $g-2$ kinematics, gives instead a much larger contribution.

The breakdown of the contribution to $a_{\mu}$ in different integration regions shows that there is in general a rather good agreement (with a few exceptions) between the Regge and the CCDGI/HW2 models. In particular in the pion $/ a_{1}$ channel, the agreement is very good in the "asymptotic" region, the first row in the table. At low $q^{2}$ there are differences, but these are expected, because the two models describe different degrees of freedom there. The situation is similar in the $\eta / f_{1}+\eta^{\prime} / f_{1}^{\prime}$ channels, where again the largest differences occur in the low-energy region. However, there are also some non-negligible differences even in the large- $Q_{i}^{2}$ region, which might be related to the fact that the HW2 models do not fully saturate the SDC2 [44]. Overall, the PS Regge and also the hQCD models are largely compatible with the LP interpolants, which are independent of the choice of degrees of freedom. As far as the MV model is concerned, all regions where at least one of the $Q_{i}$ is large are in reasonable agreement with the other two models, but it is the region where all $Q_{i}$ are small where the MV model estimates significantly larger effects; as expected, since in this region the truncation of the non-factorizable contributions, see Sect. 5.2, cannot be justified. The table also shows that the kinematic region $Q_{1}^{2} \sim Q_{2}^{2} \gg Q_{3}^{2}, Q_{1,2}^{2}>Q_{\text {match }}$, all contained in part of the first row and in the second row, provides a small contribution to the total. This is particularly true for the MV model.

Another way to visualize the impact on $a_{\mu}$ of the different kinematic regions is to plot the contribution to $a_{\mu}$ as a function of a lower cutoff $Q_{\min }<Q_{i}$, as shown in Fig. 5. The plot shows again that although there are differences in the $Q_{i}$ dependence between the Regge and the hQCD models, these are not so significant with respect to $a_{\mu}$ and lead to a similar 


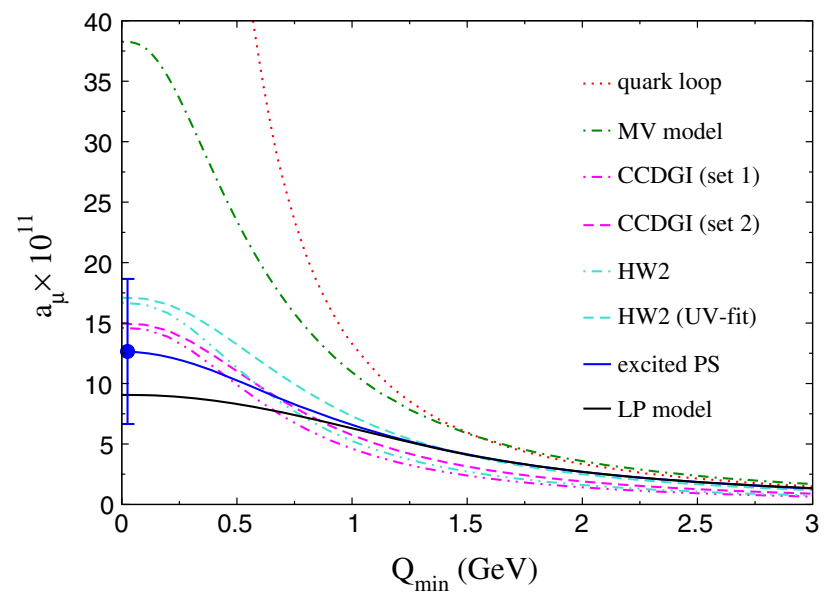

Fig. 5 Contribution to $a_{\mu}$ for $Q_{i} \geq Q_{\text {match }}$ : the longitudinal part of the massless perturbative QCD quark loop (dotted red), the MV model (dotdashed green), the CCDGI model (dashed and dotted-dashed magenta), the LR HW2 model (dashed and dotted-dashed turquoise), the LP model (solid black), and our model (solid blue). The blue point indicates the final value in (30)

final number. The MV model, on the other hand, only comes close to the other two for large values of $Q_{\min }$, whereas it estimates a much larger effect in the region of low $Q_{i}$. Finally, we have also shown the result obtained with the interpolants by LP, which is compatible with both the Regge as well as the hQCD models, even though somewhat lower for low$Q^{2}$. This may have to do with the fact that it does not include any explicit resonances and lacks the corresponding low- $Q^{2}$ enhancements. However, as explained in [45], the method of interpolants can be generalized to explicitly include resonance contributions, once their model-independent description becomes available (and might offer a valuable alternative to the resummation of a tower of states). For axial-vector states this is not yet the case, however: a phenomenologically driven evaluation seems within reach at least for the $f_{1}$ contribution [72], but it will require a detailed understanding of sum-rule ambiguities.

The present numerical comparison seems to be at odds with the conclusions drawn by CCDGI [44], who claim to be in agreement with the MV estimate. They reach this conclusion on the basis of two comparisons: a detailed one at the level of the $\langle V V A\rangle$ correlation function and one at the level of the total contribution to $a_{\mu}$. The first one has been discussed above and indeed shows that the two models agree very well. However, the comparison of the contribution to $a_{\mu}$ at the level of the total without separation of the poles of the ground-state pseudoscalars risks to be misleading: the total number is dominated by the poles due to the Goldstone bosons and even small differences in the evaluation of the latter (necessary because of our improved understanding of their TFF) may obscure the comparison for the remainder. LR [43], whose model coincides algebraically with that of

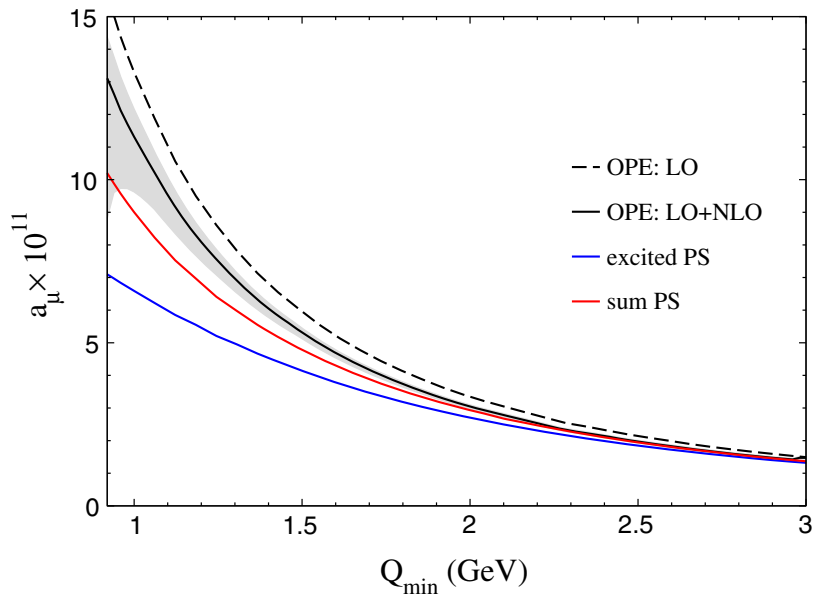

Fig. 6 Matching between the NLO OPE and the Regge model for pseudoscalars (red curve). The blue curve shows the contribution of only the excited pseudoscalars, excluding the $\pi^{0}, \eta$, and $\eta^{\prime}$. The gray band refers to the uncertainty in setting the $\alpha_{s}$ input, see [42] for more details

CCDGI and numerically differs very little, make the comparison after first subtracting the Goldstone-boson poles and come to the same conclusion we reached here.

\section{Impact of the perturbative corrections to the OPE}

We can now evaluate the effect of the recently calculated gluonic corrections to the OPE [42], as illustrated in Fig. 6. The NLO corrections lead to a reduction of the massless quark loop that for integrated quantities tends to evaluate around $1-\alpha_{s} / \pi$, e.g., one has at the symmetric point $Q_{1}=$ $Q_{2}=Q_{3}=Q[42]$

$$
\begin{aligned}
\frac{\left.\bar{\Pi}_{1}\right|_{\mathrm{NLO}}}{\left.\bar{\Pi}_{1}\right|_{\mathrm{LO}}} & =C_{1} \frac{\alpha_{s}}{\pi}, \\
C_{1} & =-\frac{75 \Delta^{(1)}-2 \Delta^{(3)}-360 \zeta_{3}}{54} \approx-0.86,
\end{aligned}
$$

where $\Delta^{(n)}=\psi^{(n)}(1 / 3)-\psi^{(n)}(2 / 3)$ in terms of the polygamma function $\psi^{(n)}$ and $\zeta_{3} \approx 1.202$ (the coefficient of the $\alpha_{s}$ corrections becomes exactly -1 in the MV limit [45]). In the following, we use the full corrections from [42].

As the hQCD models and the Regge model agree reasonably well at the numerical level, we will rely only on the latter in the following. We essentially repeat the matching procedure described in [27], replacing the plain massless quark loop with the one containing $\mathcal{O}\left(\alpha_{s}\right)$ corrections. The resulting shift, even down to momentum cutoffs of about $1 \mathrm{GeV}$ is small, as can be seen in Fig. 6, but the most relevant improvement is the reduction of the uncertainties, which had been estimated to be $\mathcal{O}(20 \%)$ of the massless quark loop in [27] and is reduced to a few percent after the NLO calculation [42], 
much smaller than the uncertainties on the hadronic side. As a consequence, the procedure to determine the matching point by minimizing the total uncertainties would not work anymore. Instead, we keep it fixed at 1.7(5) GeV as we did in [27]. With these changes, our updated estimate of the impact of longitudinal SDCs on $a_{\mu}$ reads

$$
\begin{aligned}
\Delta a_{\mu}^{\mathrm{LSDC}} & =\left[8.7(5.3)_{\text {PS-poles }}+4.2(1)_{q-\text { loop }}\right] \times 10^{-11} \\
& =13(5) \times 10^{-11}
\end{aligned}
$$

where the first number in brackets is the contribution from the region below the matching momentum of $1.7 \mathrm{GeV}$, evaluated as resummation of excited pseudoscalars, and the second from the region above $1.7 \mathrm{GeV}$, evaluated with the NLO quark loop. A welcome feature of the perturbative corrections is that they push the OPE curve down, thereby improving the matching with the hadronic model, which now seems to work perfectly already around $2 \mathrm{GeV}$.

If one takes into account the large uncertainties on the hadronic curve (not shown in Fig. 6) and the rather small estimated perturbative and non-perturbative uncertainties, one would be led to push the matching point towards $1 \mathrm{GeV}$ : this would reduce the importance of both hadronic and model uncertainties and lead to smaller total uncertainties. To illustrate the point we mention the number we get for a matching point at the lower end of the range we considered, for 1.2 GeV: $\Delta a_{\mu}^{\text {LSDC }}=\left[5.7(2.8)_{\text {PS-poles, }}\right.$ par $\left.+8.1(5)_{q \text {-loop }}\right] \times$ $10^{-11}=14(3) \times 10^{-11}$, where the error in the hadronic model only refers to the parametric uncertainty-reduced from the $3.6 \times 10^{-11}$ it contributes to (30) - while the remainder of the error estimate, especially the variation of the matching point, does not adapt in a straightforward way to the lower scale. Most notably, the central value only changes slightly, well within the uncertainties of the hadronic model, which shows that the information coming from the perturbative side agrees with the hadronic estimate. Of course, it is not obvious that at such low energies power corrections beyond the ones calculated in [41] remain irrelevant, and to be on the safe side one may consider increasing a bit the uncertainties on the OPE side (also perturbative corrections, estimated in [42] via scale variation in $\alpha_{s}$, could play a role at such low energies). In any case, this brief discussion is just meant to underline the relevance of the calculation of the corrections to the massless quark loop and their possible impact in reducing the uncertainties of this contribution: a full implementation of this is left for future work.

\section{Conclusions and outlook}

In this paper we have discussed our current understanding of the role and impact of longitudinal SDCs on the HLbL con- tribution to $(g-2)_{\mu}$ and updated it to take into account the recent calculation of the NLO perturbative corrections to the OPE [42]. On the low-energy, hadronic side different solutions for the matching to the SDCs have been proposed, sometimes accompanied by contradicting statements. To clarify the situation we have compared these models both at the level of the longitudinal component $w_{L}$ of three-point function $\langle V V A\rangle$ and in terms of the function $G$, defined in (2), which collects all contributions beyond the pion pole to the $\bar{\Pi}_{1}$ function of the HLbL tensor-the only one relevant for the longitudinal SDCs. In this way, the core assumptions and features of each implementation become most transparent, facilitating the comparison of the different proposed solutions.

Our conclusions can be summarized as follows:

1. Both the original MV model and the recent hQCD models satisfy the axial anomaly in the chiral limit exactly. When compared at the level of the three-point function and the longitudinal component $w_{L}\left(q^{2}\right)$, they agree very well, supporting that the MV model is an excellent approximation to QCD for this particular quantity. We have also compared our Regge model for excited pseudoscalars [27] and showed that, as expected, it satisfies the axial anomaly only asymptotically and at low $q^{2}$.

2. A comparison between the MV and the hQCD models for the four-point function, and in particular the function $G$, can be done analytically and is very transparent: the MV model can be viewed as a truncation of the hQCD models and amounts to dropping all contributions beyond the pion pole for $g-2$ kinematics. In the hQCD models these additional contributions are expressed in terms of a single integral over Bessel functions, which cannot be factorized into a function of $q_{1,2}^{2}$ and one of $q_{3}^{2}$. We have analyzed the relative importance of the non-factorizable and the MV term and shown that the latter is dominant only for rather large values of $q_{1,2}^{2}$ : neglecting the former term leads to a significant overestimate of the low $-q_{1,2}^{2}$ contribution. The hQCD and the MV model, which agree almost exactly on the axial anomaly, thus differ substantially in their estimate of $a_{\mu}^{\mathrm{HLbL}}$.

3. The two approaches that achieve a matching to the OPE by resumming a tower of hadronic states provide very similar estimates of the impact on $a_{\mu}$, even though one is based on excited pseudoscalars in a Regge model [27] and the other on axial-vector mesons in a hQCD model $[43,44]$, with the aforementioned differences in $w_{L}\left(q^{2}\right)$ in the transition region between low and high momenta. This again shows that the role of the axial anomaly in determining the HLbL amplitude and its contribution to $a_{\mu}$ is rather limited. 
4. The hQCD models $[43,44]$ provide an explicit, analytic solution of the SDC1 in terms of a tower of axial-vector resonances, which offers useful insights in the mechanism by which SDC1 is fulfilled. Simple versions of these models, such as HW2, depend on very few parameters, which can be pinned down by imposing a number of phenomenological constraints, but once this is done further comparisons to phenomenology show discrepancies. This can be improved by considering more complicated versions of these models, such as HW1.

5. In the chiral limit the axial-vector mesons have to play an important role in satisfying the SDCs, and the hQCD models provide a concrete realization of the underlying mechanism. In the future it will be critical to achieve a full, model-independent understanding of how axialvector resonances contribute to HLbL (in analogy to scalar states [58]), at least in the narrow-width approximation. Otherwise a combination with other contributions to HLbL scattering evaluated within a dispersive approach would not be justified. Here, we have presented the expression for the dispersive axial-vector contribution to $\bar{\Pi}_{1}$ in a particular choice of basis that is compatible with all contributions evaluated dispersively so far, but sum-rule ambiguities that are reflected in a basis dependence still need to be addressed together with a numerical analysis based on any TFF input.

6. The final estimates of $\Delta a_{\mu}^{\mathrm{LSDC}}$ obtained with the hQCD and our Regge model agree quite well with each other as well as with a solution of the SDCs based on interpolants [45]. On this basis, we have updated the final result given in [27] to incorporate the perturbative corrections to the OPE calculated in [42]:

$$
\Delta a_{\mu}^{\mathrm{LSDC}}=13(5) \times 10^{-11} .
$$

Even with the slight reduction of the total uncertainty, this covers all realistic estimates of the impact of the longitudinal SDCs on $a_{\mu}^{\mathrm{HLbL}}$ present in the literature.

As we argued above, further reductions of the uncertainties in the HLbL contribution due to the fulfillment of the SDCs are possible, also in view of the smallness of the perturbative corrections to the OPE and their uncertainties down to $1 \mathrm{GeV}$ [42]. This will require an improved and less modeldependent description on the hadronic side before trying to optimize the matching and exploiting at best the result of the perturbative calculation. Some of the recent developments discussed here have paved the way to this goal. Future steps in this direction include:

(i) fully clarifying how to evaluate the contribution of axialvector resonances to $a_{\mu}^{\mathrm{HLbL}}$ in an unambiguous way; (ii) understanding how to incorporate the solution of the SDCs in the chiral limit provided by the hQCD models in a more general, dispersively motivated framework based on axial-vector mesons;

(iii) while our discussion here was only concerned with the SDC for the longitudinal amplitude, a solution in terms of axial vectors can address at the same time both the longitudinal and the transverse SDCs;

(iv) once the treatment of axial-vector mesons in the general dispersive formalism will become possible, the reasons to use a Regge model of pseudoscalars as a tool to estimate the impact of the SDCs will cease to exist: only the few lightest excited pseudoscalars will need to be included.

Work along these lines is ongoing.

Acknowledgements We thank Jan Lüdtke and Massimiliano Procura for discussions and for providing numbers about their model. We further thank Antonio Rodríguez-Sánchez for help with the numerical evaluation of the perturbative correction to the OPE, and Luigi Cappiello, Oscar Catà, Giancarlo D'Ambrosio, Nils Hermansson-Truedsson, Abhishek Iyer, Marc Knecht, Josef Leutgeb, Jan Lüdtke, Massimiliano Procura, and Anton Rebhan for comments on the manuscript. Financial support by the SNSF (Project Nos. PCEFP2_181117 and PZ00P2_193383 and Grant No. 200020_175791) is gratefully acknowledged.

Data Availability Statement This manuscript has no associated data or the data will not be deposited. [Authors' comment: There is no data because the work is of theoretical nature.]

Open Access This article is licensed under a Creative Commons Attribution 4.0 International License, which permits use, sharing, adaptation, distribution and reproduction in any medium or format, as long as you give appropriate credit to the original author(s) and the source, provide a link to the Creative Commons licence, and indicate if changes were made. The images or other third party material in this article are included in the article's Creative Commons licence, unless indicated otherwise in a credit line to the material. If material is not included in the article's Creative Commons licence and your intended use is not permitted by statutory regulation or exceeds the permitted use, you will need to obtain permission directly from the copyright holder. To view a copy of this licence, visit http://creativecomm ons.org/licenses/by/4.0/.

Funded by SCOAP ${ }^{3}$.

\section{References}

1. B. Abi et al., [Muon $g-2$ Collaboration], Phys. Rev. Lett. 126, 141801 (2021). arXiv:2104.03281 [hep-ex]

2. T. Albahri et al., [Muon $g-2$ Collaboration], Phys. Rev. D 103, 072002 (2021). arXiv:2104.03247 [hep-ex]

3. T. Albahri et al., [Muon $g-2$ Collaboration], Phys. Rev. A 103, 042208 (2021). arXiv:2104.03201 [hep-ex]

4. T. Albahri et al., [Muon $g-2$ Collaboration], Phys. Rev. Accel. Beams 24, 044002 (2021). arXiv:2104.03240 [physics.acc-ph]

5. T. Aoyama et al., Phys. Rep. 887, 1 (2020). arXiv:2006.04822 [hep-ph] 
6. T. Aoyama, M. Hayakawa, T. Kinoshita, M. Nio, Phys. Rev. Lett. 109, 111808 (2012). arXiv:1205.5370 [hep-ph]

7. T. Aoyama, T. Kinoshita, M. Nio, Atoms 7, 28 (2019)

8. A. Czarnecki, W.J. Marciano, A. Vainshtein, Phys. Rev. D 67, 073006 (2003). arXiv:0212229 [hep-ph] [Erratum: Phys. Rev. D 73, 119901 (2006)]

9. C. Gnendiger, D. Stöckinger, H. Stöckinger-Kim, Phys. Rev. D 88, 053005 (2013). arXiv:1306.5546 [hep-ph]

10. M. Davier, A. Hoecker, B. Malaescu, Z. Zhang, Eur. Phys. J. C 77, 827 (2017). arXiv: 1706.09436 [hep-ph]

11. A. Keshavarzi, D. Nomura, T. Teubner, Phys. Rev. D 97, 114025 (2018). arXiv:1802.02995 [hep-ph]

12. G. Colangelo, M. Hoferichter, P. Stoffer, JHEP 02, 006 (2019). arXiv:1810.00007 [hep-ph]

13. M. Hoferichter, B.-L. Hoid, B. Kubis, JHEP 08, 137 (2019). arXiv:1907.01556 [hep-ph]

14. M. Davier, A. Hoecker, B. Malaescu, Z. Zhang, Eur. Phys. J. C 80, 241 (2020). arXiv:1908.00921 [hep-ph] [Erratum: Eur. Phys. J. C $80,410(2020)]$

15. A. Keshavarzi, D. Nomura, T. Teubner, Phys. Rev. D 101, 014029 (2020). arXiv:1911.00367 [hep-ph]

16. B.-L. Hoid, M. Hoferichter, B. Kubis, Eur. Phys. J. C 80, 988 (2020). arXiv:2007.12696 [hep-ph]

17. A. Kurz, T. Liu, P. Marquard, M. Steinhauser, Phys. Lett. B 734, 144 (2014). arXiv:1403.6400 [hep-ph]

18. K. Melnikov, A. Vainshtein, Phys. Rev. D 70, 113006 (2004). arXiv:hep-ph/0312226

19. P. Masjuan, P. Sánchez-Puertas, Phys. Rev. D 95, 054026 (2017). arXiv: 1701.05829 [hep-ph]

20. G. Colangelo, M. Hoferichter, M. Procura, P. Stoffer, Phys. Rev. Lett. 118, 232001 (2017). arXiv:1701.06554 [hep-ph]

21. G. Colangelo, M. Hoferichter, M. Procura, P. Stoffer, JHEP 04, 161 (2017). arXiv:1702.07347 [hep-ph]

22. M. Hoferichter, B.-L. Hoid, B. Kubis, S. Leupold, S.P. Schneider, Phys. Rev. Lett. 121, 112002 (2018). arXiv:1805.01471 [hep-ph]

23. M. Hoferichter, B.-L. Hoid, B. Kubis, S. Leupold, S.P. Schneider, JHEP 10, 141 (2018). arXiv:1808.04823 [hep-ph]

24. A. Gérardin, H.B. Meyer, A. Nyffeler, Phys. Rev. D 100, 034520 (2019). arXiv:1903.09471 [hep-lat]

25. J. Bijnens, N. Hermansson-Truedsson, A. Rodríguez-Sánchez, Phys. Lett. B 798, 134994 (2019). arXiv:1908.03331 [hep-ph]

26. G. Colangelo, F. Hagelstein, M. Hoferichter, L. Laub, P. Stoffer, Phys. Rev. D 101, 051501 (2020). arXiv:1910.11881 [hep-ph]

27. G. Colangelo, F. Hagelstein, M. Hoferichter, L. Laub, P. Stoffer, JHEP 03, 101 (2020). arXiv:1910.13432 [hep-ph]

28. T. Blum, N. Christ, M. Hayakawa, T. Izubuchi, L. Jin, C. Jung, C. Lehner, Phys. Rev. Lett. 124, 132002 (2020). arXiv:1911.08123 [hep-lat]

29. G. Colangelo, M. Hoferichter, A. Nyffeler, M. Passera, P. Stoffer, Phys. Lett. B 735, 90 (2014). arXiv:1403.7512 [hep-ph]

30. G.W. Bennett et al., Muon $g-2$ Collaboration, Phys. Rev. D 73, 072003 (2006). arXiv:hep-ex/0602035

31. J. Grange et al., [Muon $g-2$ Collaboration], arXiv:1501.06858 [physics.ins-det]

32. M. Abe et al., PTEP 2019, 053 C02 (2019). arXiv:1901.03047 [physics.ins-det]

33. E.-H. Chao, R.J. Hudspith, A. Gérardin, J.R. Green, H.B. Meyer, K. Ottnad, Eur. Phys. J. C 81, 651 (2021). arXiv:2104.02632 [hep-lat]

34. S.L. Adler, Phys. Rev. 177, 2426 (1969)

35. J.S. Bell, R. Jackiw, Nuovo Cim. A 60, 47 (1969)

36. W.A. Bardeen, Phys. Rev. 184, 1848 (1969)

37. J. Wess, B. Zumino, Phys. Lett. B 37, 95 (1971)

38. E. Witten, Nucl. Phys. B 223, 422 (1983)

39. A. Vainshtein, Phys. Lett. B 569, 187 (2003). arXiv:hep-ph/0212231
40. M. Knecht, S. Peris, M. Perrottet, E. de Rafael, JHEP 03, 035 (2004). arXiv:hep-ph/0311100

41. J. Bijnens, N. Hermansson-Truedsson, L. Laub, A. RodríguezSánchez, JHEP 10, 203 (2020). arXiv:2008.13487 [hep-ph]

42. J. Bijnens, N. Hermansson-Truedsson, L. Laub, A. RodríguezSánchez, JHEP 04, 240 (2021). arXiv:2101.09169 [hep-ph]

43. J. Leutgeb, A. Rebhan, Phys. Rev. D 101, 114015 (2020). arXiv:1912.01596 [hep-ph]

44. L. Cappiello, O. Catà, G. D'Ambrosio, D. Greynat, A. Iyer, Phys. Rev. D 102, 016009 (2020). arXiv:1912.02779 [hep-ph]

45. J. Lüdtke, M. Procura, Eur. Phys. J. C 80, 1108 (2020). arXiv:2006.00007 [hep-ph]

46. S. Peris, M. Perrottet, E. de Rafael, JHEP 05, 011 (1998). arXiv:hep-ph/9805442

47. J. Bijnens, E. Gámiz, E. Lipartia, J. Prades, JHEP 04, 055 (2003) arXiv:hep-ph/0304222

48. M. Hoferichter, G. Colangelo, M. Procura, P. Stoffer, Int. J. Mod. Phys. Conf. Ser. 35, 1460400 (2014). arXiv:1309.6877 [hep-ph]

49. G. Colangelo, M. Hoferichter, M. Procura, P. Stoffer, JHEP 09, 091 (2014). arXiv:1402.7081 [hep-ph]

50. G. Colangelo, M. Hoferichter, B. Kubis, M. Procura, P. Stoffer, Phys. Lett. B 738, 6 (2014). arXiv:1408.2517 [hep-ph]

51. G. Colangelo, M. Hoferichter, M. Procura, P. Stoffer, JHEP 09, 074 (2015). arXiv:1506.01386 [hep-ph]

52. K. Melnikov, A. Vainshtein, arXiv:1911.05874 [hep-ph]

53. J. Bijnens, J. Prades, Mod. Phys. Lett. A 22, 767 (2007). arXiv:hep-ph/0702170

54. M. Knecht, JHEP 08, 056 (2020). arXiv:2005.09929 [hep-ph]

55. G.P. Lepage, S.J. Brodsky, Phys. Lett. B 87, 359 (1979)

56. G.P. Lepage, S.J. Brodsky, Phys. Rev. D 22, 2157 (1980)

57. P. Masjuan, P. Roig, P. Sánchez-Puertas, arXiv:2005.11761 [hep$\mathrm{ph}]$

58. I. Danilkin, M. Hoferichter, P. Stoffer, Phys. Lett. B 820, 136502 (2021). arXiv:2105.01666 [hep-ph]

59. P. Roig, P. Sánchez-Puertas, Phys. Rev. D 101, 074019 (2020). arXiv:1910.02881 [hep-ph]

60. V. Pauk, M. Vanderhaeghen, Eur. Phys. J. C 74, 3008 (2014). arXiv: 1401.0832 [hep-ph]

61. F. Jegerlehner, Springer Tracts Mod. Phys. 274, 1 (2017)

62. R. García-Martín, B. Moussallam, Eur. Phys. J. C 70, 155 (2010). arXiv: 1006.5373 [hep-ph]

63. M. Hoferichter, D.R. Phillips, C. Schat, Eur. Phys. J. C 71, 1743 (2011). arXiv:1106.4147 [hep-ph]

64. B. Moussallam, Eur. Phys. J. C 73, 2539 (2013). arXiv:1305.3143 [hep-ph]

65. I. Danilkin, M. Vanderhaeghen, Phys. Lett. B 789, 366 (2019). arXiv: 1810.03669 [hep-ph]

66. M. Hoferichter, P. Stoffer, JHEP 07, 073 (2019). arXiv: 1905.13198 [hep-ph]

67. I. Danilkin, O. Deineka, M. Vanderhaeghen, Phys. Rev. D 101, 054008 (2020). arXiv:1909.04158 [hep-ph]

68. I. Danilkin, O. Deineka, M. Vanderhaeghen, Phys. Rev. D 96, 114018 (2017). arXiv:1709.08595 [hep-ph]

69. J. Lu, B. Moussallam, Eur. Phys. J. C 80, 436 (2020). arXiv:2002.04441 [hep-ph]

70. M. Hoferichter, P. Stoffer, JHEP 05, 159 (2020). arXiv:2004.06127 [hep-ph]

71. P. Achard et al., L3 Collaboration, Phys. Lett. B 526, 269 (2002). arXiv:hep-ex/0110073

72. M. Zanke, M. Hoferichter, B. Kubis, JHEP 07, 106 (2021). arXiv:2103.09829 [hep-ph]

73. L. Gan, B. Kubis, E. Passemar, S. Tulin, arXiv:2007.00664 [hep$\mathrm{ph}]$

74. P. Achard et al., [L3 Collaboration], JHEP 03, 018 (2007) 